\title{
Papers
}

\section{The effect of specimen processing delay on borate urine preservation}

\author{
T Gillespie, J Fewster, R G Masterton
}

\begin{abstract}
Aim-To investigate the effect on urine culture results and their clinical interpretation of delaying the processing of urine samples in which boric acid had been used as a preservative.

Methods-792 mid-stream specimens of urine from patients attending their general practitioner were received in borate containing plastic jars. The specimens were cultured upon receipt, stored at room temperature, and then recultured the following morning.

Results -After overnight delayed culture, the results were altered in $16 \%$ of samples and the clinical interpretation of these findings differed in $8 \%$ of specimens. In 28 samples $(3.5 \%)$ the bacterium isolated on initial culture was not the same as that obtained by culture after overnight storage. Conclusions-Boric acid urine preservation used for overnight delayed processing of samples is associated with a significant alteration in culture results and the attendant clinical interpretation of such specimens. Rapid transportation/processing of urine specimens must remain the optimum procedure.

(F Clin Pathol 1999;52:95-98)
\end{abstract}

Keywords: boric acid preservation; urine storage; urine culture

The optimum investigation of a suspected urinary tract infection requires the culture of an appropriate specimen collected by a method which reduces, as far as possible, contamination by bacteria from the perineum. Conventionally, the diagnosis relies on culturing a pure growth of a bacterial species at a concentration of greater than or equal to $10^{5}$ colony forming units $(\mathrm{cfu}) / \mathrm{ml}^{1}{ }^{1}$ In some circumstances this value may be $10^{4} \mathrm{cfu} / \mathrm{ml}$ or less and still be significant. ${ }^{1}$ Urine is an excellent culture medium for bacteria and hence the sample should be examined as soon as possible after collection or should be refrigerated if a delay of up to 24 hours is anticipated. ${ }^{1}$ As these procedures are not always possible in general practice, an effective method of preserving urine at room temperature is desirable. The optimum method should maintain the urine at the condition of its collection-that is, con- taminants should not be able to overgrow, and pathogenic bacteria should be preserved in their original numbers and should not be inhibited in subsequent culture growth. Since Porter and Brodie ${ }^{2}$ first introduced boric acid as a method of preserving urine during storage at room temperature, many laboratories have incorporated-primarily for community specimens-a system in which sterile plastic universal containers are supplied with a measured amount of boric acid crystals. To collect the specimen the containers are charged with $15 \mathrm{ml}$ of urine to produce a final concentration of $18 \mathrm{~g} /$ litre borate ion when the crystals dissolve. Previous studies have investigated the effect of borate ion on bacteria in both urine and culture broth, principally under experimental laboratory conditions. ${ }^{34}$

In addition to technical issues, many laboratories have, for operational reasons, addressed cost pressures by exploring the possibility of rescheduling work from its arrival to a different time. The use of borate ion to preserve urine samples offers the potential to delay undertaking work on specimens collected late in the day until the following morning, with a resultant saving in out of hours staff costs. As there is no previous assessment of this application of urine containers supplied with boric acid crystals, we undertook a prospective study to investigate this use of the system.

Table 1 Details of samples excluded from the study

\begin{tabular}{lc}
\hline Reason for exclusion & Number \\
\hline Time of collection unknown, or $>8$ hours since & \\
collection & 152 \\
Catheter specimens of urine & 29 \\
Volume $<10 \mathrm{ml}$ & 84 \\
Age of patient $<16$ years (or not stated) & 143 \\
Total & 408 \\
\hline
\end{tabular}

Table 2 Clinical indications for specimen referral

\begin{tabular}{lcl}
\hline Indication for referral & Number & (\%) \\
\hline Suspected lower urinary tract infection & 524 & $(66.2)$ \\
Screening for urinary tract infection as a & & \\
$\quad$ contributing factor to other disease, eg & & \\
$\quad$ diabetes, pyrexia of unknown origin, & & \\
$\quad$ acute confusional states & 130 & $(16.4)$ \\
Antenatal & 78 & $(9.8)$ \\
Post-treatment & 59 & $(7.4)$ \\
Suspected pyelonephritis & 1 & $(0.1)$ \\
Total & 792 & $(100)$ \\
\hline
\end{tabular}

Accepted for publication 3 September 1998 
Table 3 Comparison of culture results when processed within eight hours of specimen collection and after overnight storage in the laboratory

\begin{tabular}{|c|c|c|c|c|c|c|c|}
\hline \multirow{2}{*}{$\begin{array}{l}\text { Growth (cfu/ml) } \\
\text { Cultured } \leqslant 8 \\
\text { hours }\end{array}$} & \multicolumn{6}{|c|}{ Growth (cfu/ml)—cultured after overnight storage } & \multirow[b]{2}{*}{ Total } \\
\hline & No growth & $<10^{2}$ & $10^{2}-10^{3}$ & $10^{3}-10^{4}$ & $10^{4}-10^{5}$ & $>10^{5}$ & \\
\hline No growth & 176 & 50 & 0 & 0 & 7 & 5 & 238 \\
\hline$<10^{2}$ & 56 & 169 & 0 & 5 & 26 & 12 & 268 \\
\hline $10^{2}-10^{3}$ & 0 & 0 & 0 & 0 & 0 & 0 & 0 \\
\hline $10^{3}-10^{4}$ & 1 & 2 & 1 & 3 & 2 & 1 & 10 \\
\hline $10^{4}-10^{5}$ & $\begin{array}{l}1 \\
5\end{array}$ & 20 & 0 & 4 & 62 & 21 & 112 \\
\hline$>10^{5}$ & 0 & 5 & 0 & 0 & 33 & 126 & 164 \\
\hline Total & 238 & 246 & 1 & 12 & 130 & 165 & 792 \\
\hline
\end{tabular}

Values are the number of specimens in each culture category.

Table 4 Interpretation of culture results when processed within eight hours of specimen collection and after overnight storage in the laboratory

\begin{tabular}{lrrrrr}
\hline \multirow{5}{*}{$\begin{array}{l}\text { Interpretation, } \\
\text { day 1 }\end{array}$} & \multicolumn{4}{l}{ Interpretation, day 2} & \\
\cline { 2 - 5 } & 1 & 2 & 3 & 4 & Total \\
\hline 1 & 451 & 12 & 12 & 31 & 506 \\
2 & 5 & 105 & 19 & 9 & 138 \\
3 & 6 & 6 & 18 & 12 & 42 \\
4 & 22 & 9 & 10 & 65 & 106 \\
Total & 484 & 132 & 59 & 117 & 792
\end{tabular}

The interpretation of the culture result was defined as follows: (1): no growth and no significant growth $\left(<10^{4} \mathrm{cfu} / \mathrm{ml}\right.$ in pure growth or $<10^{5} \mathrm{cfu} / \mathrm{ml}$ mixed organisms); (2) significant growth $\left(\geqslant 10^{5} \mathrm{cfu} / \mathrm{ml}\right.$ pure growth); (3) equivocal growth $\left(10^{4}-10^{5}\right.$ $\mathrm{cfu} / \mathrm{ml}$ pure growth); (4) contamination $\left(\geqslant 10^{5} \mathrm{cfu} / \mathrm{ml}\right.$ mixed organisms).

\section{Methods}

Consecutive samples of urine $(n=1175)$ in universal jars containing crystalline boric acid (Bibby Sterilin) were received from general practices served by our laboratory. Of these, 408 samples were excluded from the study, for reasons listed in table 1 . The patients were 643 females and 149 males. The clinical information on the request forms accompanying the samples was assigned by a clinical microbiologist (RGM) into one of the categories listed in table 2 .

All samples were processed by the same Medical Laboratory Scientific Officer, using

Table 5 Gross clinical interpretation of culture results before and after overnight storage

\begin{tabular}{lllll}
\hline & \multicolumn{2}{l}{ Culture after overnight storage } & \\
\cline { 3 - 4 } & & Significant & Non-significant & \multirow{2}{*}{ Total } \\
\hline \multirow{2}{*}{ Culture $\leqslant 8 h$} & Significant & 105 & 33 & 138 \\
& Non-significant & 27 & 627 & 654 \\
& Total & 132 & 660 & 792
\end{tabular}

*Includes no growth, no significant growth, equivocal growth, and contamination (see table 4). Odds ratio $=73.89$; exact $95 \%$ confidence interval $=41.28$ to 133.01 .

Table 6 Bacteria isolated when specimen processed within eight hours of collection and after overnight storage in the laboratory

\begin{tabular}{lcc}
$\begin{array}{l}\text { Identity of organism isolated in significant } \\
\text { numbers }\end{array}$ & $\begin{array}{l}\text { Number of samples positive } \\
\text { within } 8 \text { hours of collection }\end{array}$ & $\begin{array}{l}\text { Number of samples positive } \\
\text { after overnight storage }\end{array}$ \\
\hline Escherichia coli & 117 & 113 \\
Klebsiella sp & 8 & 7 \\
Pseudomonas aeruginosa & 4 & 4 \\
Proteus sp & 5 & 7 \\
Enterobacter sp & 3 & 4 \\
Citrobacter sp & 1 & 1 \\
Lactose fermenting coliforms & 4 & 2 \\
Non-lactose fermenting coliforms & 2 & 1 \\
Group B streptococcus & 12 & 12 \\
Enterococcus sp & 8 & 19 \\
Staphylococcus aureus & 3 & 3 \\
a-Haemolytic streptococcus & 5 & 2 \\
Staphylococcus saprophyticus & 2 & 2 \\
Coagulase negative staphylococcus & 2 & 1 \\
Diphtheroid & 1 & 3 \\
\hline
\end{tabular}

sterile techniques throughout. The volume of the sample was recorded and the time from sampling to processing noted. All samples included in the study were cultured for the first time within eight hours of specimen collection. Thereafter, every sample was kept at room temperature overnight (between $10^{\circ} \mathrm{C}$ and $25^{\circ} \mathrm{C}$ ) and then recultured the following morning. The results of microscopy were not recorded.

The specimens were processed by a semiquantitative method for the detection of bacteria. ${ }^{5}$ After overnight incubation on CLED agar (Unipath) in air at $35^{\circ} \mathrm{C}$, the number of colonies growing was counted and the concentration of organisms in the original sample was calculated using the relevant nomogram. ${ }^{5}$ The culture plates resulting from immediate processing and those which had been cultured after overnight storage were read independently of each other.

Bacterial culture results were recorded according to the colony counts observed. No growth, growth of $<10^{4}$ organisms $/ \mathrm{ml}$, and mixed growth at any concentration were not regarded as significant. Growth of between $10^{4}$ and $10^{5} \mathrm{cfu} / \mathrm{ml}$ in pure culture was regarded as equivocal. A result of greater than or equal to $10^{5} \mathrm{cfu} / \mathrm{ml}$ in pure culture was assessed as being significant and indicating bacterial infection. All organisms from growth assessed as significant or equivocal were identified to species level using standard microbiological methods. ${ }^{6}$

The computer software package EPI-INFO (Version 5, Centre for Disease Control, Epidemiology Program Office, Atlanta, Georgia, USA) was used to analyse the results. The odds ratio was used to determine the statistical significance of the results.

\section{Results}

A comparison between the concentration of bacterial growth when cultures were processed within eight hours of collection and the culture result from the same specimen after overnight storage is shown in table 3 . There was inhibition of growth in 127 samples (16\%), and increased growth in 129 samples (16.2\%) when culture was delayed by overnight storage.

In table 4, account is taken of whether the bacterial growth was mixed or a pure culture. A comparison of the clinical microbiological assessment of the culture findings is given in table 5. The difference in the number of samples where the interpretation changed from a significant to a non-significant culture result after overnight storage and the number of samples where the interpretation altered from a non-significant to a significant culture result was clinically significant (odds ratio $=73.89$; 95\% confidence interval, 41.28 to 133.01 ). Although a slight increase from 106 to 117 in the number of samples recorded as contaminated was also observed, this does not achieve statistical significance (odds ratio $=0.89 ; 95 \%$ confidence interval, 0.66 to 1.20 ). The identity of the significant and equivocal isolates is shown in table 6. 


\section{Discussion}

Recommended methods to preserve the sample in the same condition as at the time of collection include refrigeration and the addition to the urine of substances which are inhibitors of bacterial growth. Ideally, a sample of urine sent for investigation of infection should be examined and cultured within four hours of collection, ${ }^{7}$ as the length of time before significant bacterial multiplication occurs may be as short as 90 minutes. ${ }^{8}$ However, current clinical practice, particularly for samples received from the community, dictates that there is often several hours delay between specimen collection and processing. General practitioners may run surgeries which extend later than the normal laboratory working day, and transport services to the laboratory may not always be optimal. We have noticed an increasing proportion of samples arriving as evening deliveries. These require additional and later processing if they are to be dealt with on the day of receipt. With ever greater constraints on laboratory resources, a system which allows a delay in processing late specimens until the next normal working day would offer potential savings to the laboratory. Such a delay may be clinically acceptable in processing urine samples, as treatment is often instigated empirically. Obviously, any change in practice which increases the time taken to produce a result must not compromise diagnostic performance or clinical relevance.

Lum and Meers previously compared culture of urine specimens 24 and 48 hours after receipt, with and without borate ion as a preservative. ${ }^{8}$ Their study investigated hospital samples of unspecified type, age, and clinical indication under storage conditions in a laboratory in a tropical country (ambient temperature $\sim 25^{\circ} \mathrm{C}$ ). They showed an increase in bacteriologically significant growth indicating infection $(17 \%$ to $21 \% ; \mathrm{p}>0.05)$ at 48 hours in samples preserved with borate ion, compared with a statistically significant rise $(22 \%$ to $62 \%$ ) among unpreserved samples over the same time. They concluded that borate ion was effective as a urine preservative in their laboratory conditions.

Our study aimed to explore aspects relevant to current United Kingdom practice and investigated whether an overnight delay before processing a borate containing sample of urine would affect the culture results in environmental conditions experienced in a temperate country. In an attempt to produce a clear clinical conclusion, our work, which analysed a much larger sample size $(\mathrm{n}=792 v 175)$, focused on a single type of urine sample from patients with defined clinical conditions. Other steps were also taken to try to avoid confounding factors. All the specimens were dealt with by the same laboratory worker and they were only processed if they were received within eight hours of collection. Also, the samples studied were restricted to those containing between 10 and $25 \mathrm{ml}$ of urine, in order that the borate concentration lay between 10.8 and $27 \mathrm{~g} /$ litre. Despite laboratory guidelines to our users specifying the appropriate sample volume, $9.6 \%$ of tests were forwarded to the labo- ratory with calculated borate concentrations in excess of $27 \mathrm{~g} /$ litre. At such levels, bacterial inhibition would be more likely. Paediatric samples were excluded from this study as it is difficult to obtain an appropriate urine volume from young children.

The influence of the effect of the addition of boric acid crystals to a final concentration of $18 \mathrm{~g} /$ litre borate ion as a means of preventing bacterial overgrowth upon delaying the processing of urine samples has been studied both in specimen laboratory storage and in transportation..$^{2-4} 7$ An earlier in vitro study showed that the effect of a concentration of borate ion between 10 and $20 \mathrm{~g} /$ litre is bacteriostatic. However, at a higher concentration of $40 \mathrm{~g} /$ litre borate ion, and with exposure times greater than six hours, the effect can becomes inhibitory, for example to Streptococcus agalactiae, Acinetobacter calcoaceticus, and Pseudomonas aeruginosa, when reductions in bacterial concentration can occur. ${ }^{3}$ Even at the recommended borate ion concentrations, toxic effects on some of the common urinary pathogens have been recorded ${ }^{4}$ with coliform organisms reported as being inhibited more than enterococci. ${ }^{239}$ This may explain in part the altered identification of the isolates we obtained between early and delayed culture, where the number of enterococci isolated more than doubled despite more minor changes among Gram negative organisms. Obviously, any changes in identification can have important clinical implications involving diagnosis and treatment, including the conclusions reached from samples sent after treatment.

While Lum and Meers, investigating the effect of borate preservation and delayed processing under tropical conditions, did not report a significant increase in the percentage of samples regarded as being culture positive after storage at $25^{\circ} \mathrm{C}$ for 48 hours, their results showed a trend in that direction. ${ }^{8}$ Our results, involving a much larger sample size in a temperate climate, were statistically significant. In $16 \%$ of the specimens tested (127 of 792), the bacterial concentration was altered after overnight culture delay. As a result, interpretation of the culture result changed in $8 \%$ of cases (60 of 792). The interpretation altered from significance to non-significance (33 occasions) and vice versa (27 occasions). This has obvious implications for patient management.

Our study indicates that a laboratory in the United Kingdom which uses boric acid crystals as a preservative to permit an overnight delay in processing mid-stream samples of urine may produce a significantly altered range of results from those that would have been achieved if culture had been performed on the day of sample receipt. Hence our results raise questions about the validity of the earlier, less defined, investigations that were used to substantiate the role of boric acid crystals as a urine preservative. These former studies were characterised by small sample sizes spread across all age groups, sample types, and ages of specimens, where borate ion concentration was not 
assessed, and where samples were being forwarded for the investigation of clinical conditions that were not well categorised. Further work into other clinical settings is required before preservation using boric acid crystals can be universally recommended. Until then the optimum situation must remain a rapid method of transporting the urine sample from the patient to the laboratory, followed by prompt processing upon its arrival.

1 Shanson DC. Infections of the urinary tract. In: Microbiology in clinical practice, 2nd edition. Sevenoaks: John Wright, 1989:430-51.

2 Porter IA, Brodie J. Boric acid preservation of urine samples. BMF 1969;ii:353-5.
3 Meers PD, Chow CK. Bacteriostatic and bactericidal actions of boric acid against bacteria and fungi commonly found in urine. $\mathcal{F}$ Clin Pathol 1990;43:484-7.

4 Watson PG, Duerden BI. Laboratory assessment of physical and chemical method for preserving urine samples. $\mathcal{F}$ Clin Pathol 1977;30:532-6.

5 Urquhart GED, Gould JC. Simplified technique for counting the number of bacteria in urine and other fluids. $\mathcal{F} \mathrm{Clin}$ Pathol 1965;18:480-1.

6 Barrow GI, Feltham RKA, eds. Cowan Eீ Steel's manual for the identification of medical bacteria, 3rd edition. Cambridge: Cambridge University Press, 1993.

7 Wheldon DB, Slack M. Multiplication of contaminant bacteria in urine and interpretation of delayed culture. $\mathcal{f}$ Clin Pathol 1977;30:615-19.

8 Lum KT, Meers PD. Boric acid converts urine into an effective bacteriostatic transport medium. F Infect 1989;18:51-8.

9 Nickander KK, Shanholtzer CJ, Peterson LR. Urine culture transport tubes: effect of sample volume on bacterial toxic ity of the preservative. $\mathcal{F}$ Clin Microbiol 1982;15:593-5. 